\title{
Effect of Environment Enrichment (SPARK Perceptual-Motor Exercises) on the Improvement of Neurocognitive Functions in Children with Developmental Coordination Disorder
}

\author{
Hadi Moradi $^{*}$, Ahmadreza Movahedi \\ ${ }^{1}$ Department of Motor Behavior, Faculty of Sport Sciences, Ferdowsi University of Mashhad, Mashhad, Iran \\ ${ }^{2}$ Department of Motor Behavior, Faculty of Sport Sciences, University of Isfahan, Isfahan, Iran
}

Article Info:

Received: 27 Jul 2018

Revised: 26 Dec 2018

Accepted: 7 May 2019

\section{A BSTRACT}

Introduction: Developmental Coordination Disorder (DCD) is a serious childhood disorder that causes social, emotional, cognitive, and motor difficulties for children. Accordingly, the current study aimed to examine the effect of perceptual-motor training on the improvement of neurocognitive functions in children with DCD. Materials and Methods: Twenty children were selected through simple random sampling from the children with DCD. The participants were classified into the experimental (10 children) and control (10 children) groups. The children in the experimental group underwent perceptual-motor training for 8 weeks (24 sessions). The neurocognitive functions of attention were assessed in the pretest and posttest using Conners' Neuropsychological Scale (the Conners 3). Results: The results showed a significant difference between the mean neurocognitive functions of attention in the experimental and control groups. These functions improved considerably in the children of the experimental group. Conclusion: The results indicated that perceptual-motor training can be considered as a useful intervention technique to improve the neurocognitive functions of attention in children with DCD.

Key words:

1. Exercise

2. Attention

3. Child

*Corresponding Author: Hadi Moradi

E-mail: hadi.moradi@stu.um.ac.ir 


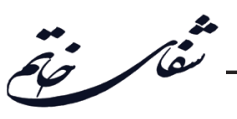

تأثير غنىسازى محيط (تمرينات ادراكى -حركتى اسيارك) بر بهبود كاركردهاى عصبى -شناختى توجه

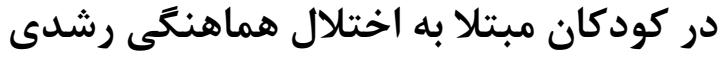

\section{هادى مر ادى"'، احمدرضا موحدى'}

اكروه رفتار حركتى، دانشكده علوم ورزشى، دانشعاه فردوسى مشهيد، مشهد، ايران

rكروه رفتار حركتى، دانشكده علوم ورزشى، دانشكاه اصفهان، اصفهان، ايران

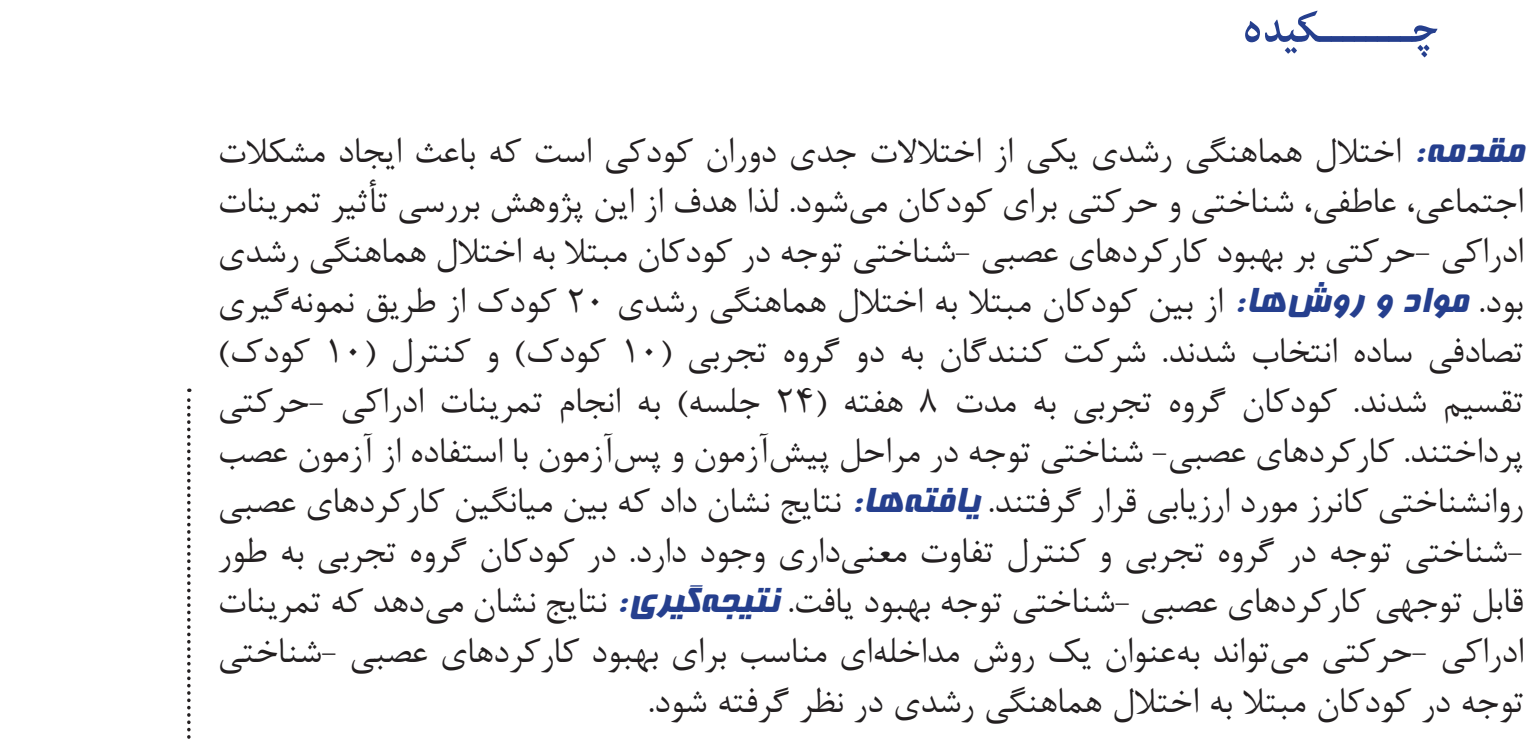

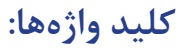

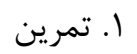

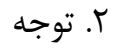

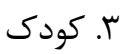

" نويسنده مسئول: هادى مرادى

آدرس الكترونيكى: hadi.moradi@stu.um.ac.ir 


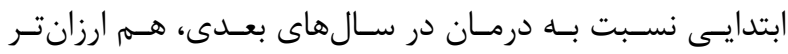

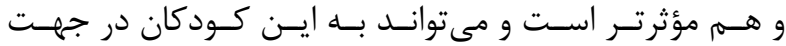

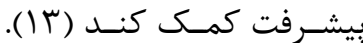

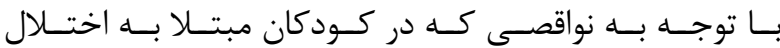

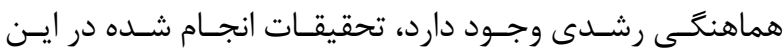

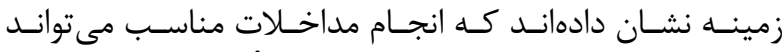

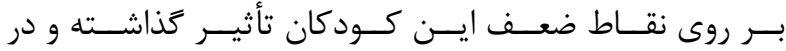

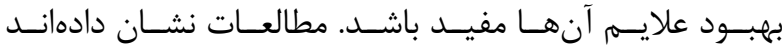

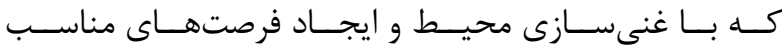

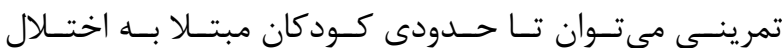

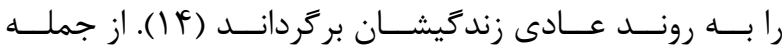

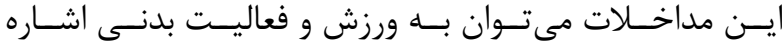

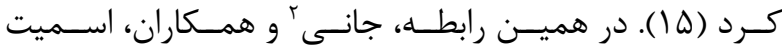

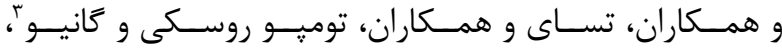

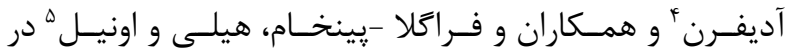

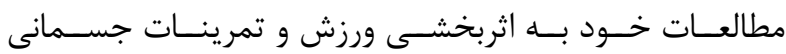

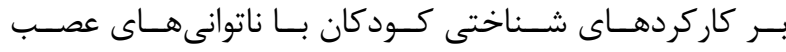

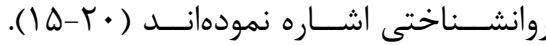

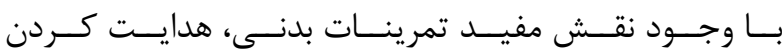

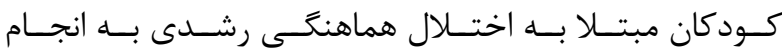

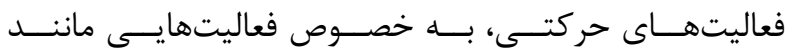

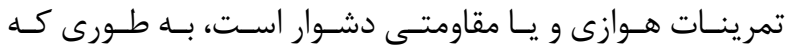

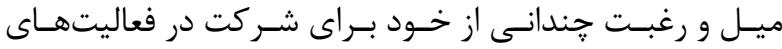

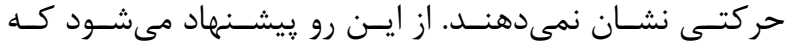

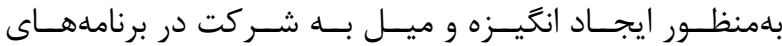

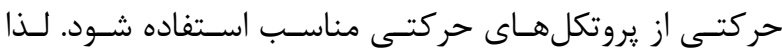

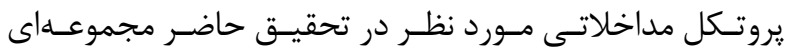

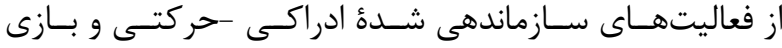

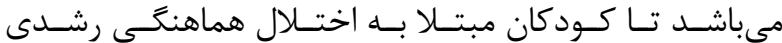

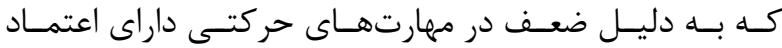

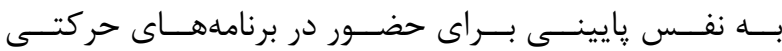

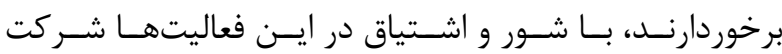

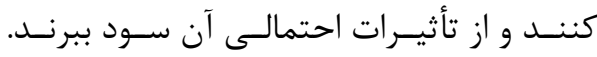

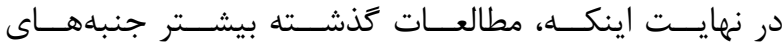

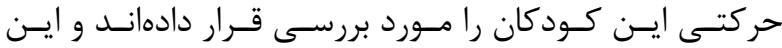

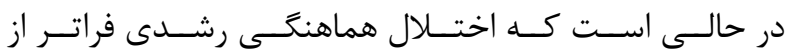

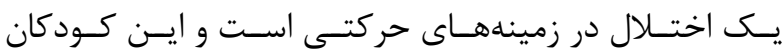

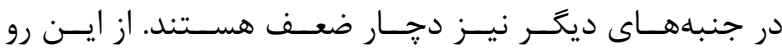

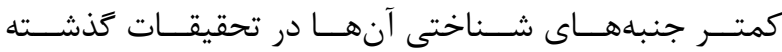

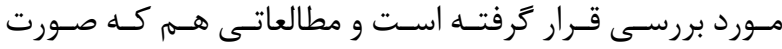

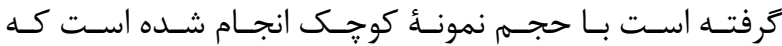

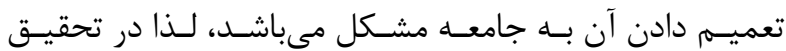

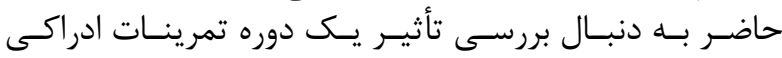

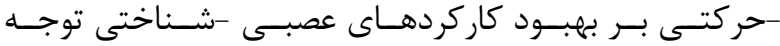

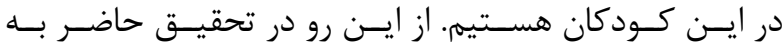

${ }^{1}$ Developmental coordination disorders

2 Jane

${ }^{3}$ Tompo, Rowski and Ganio

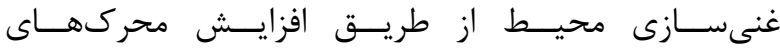

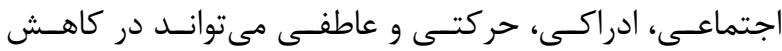

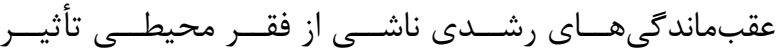

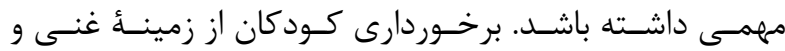

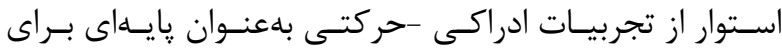

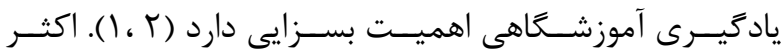

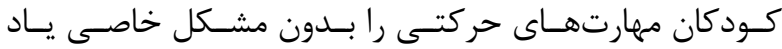

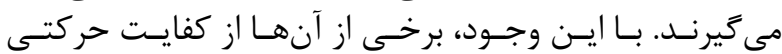

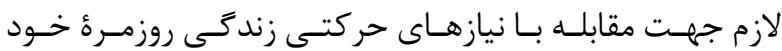

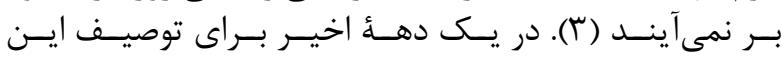

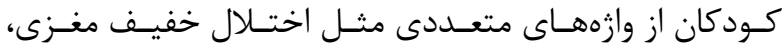

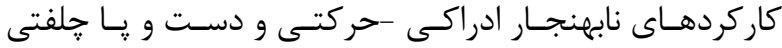

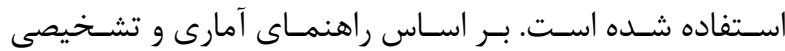

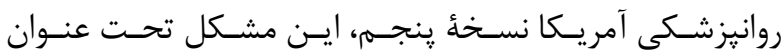

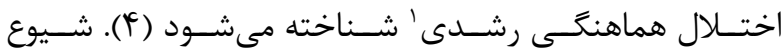

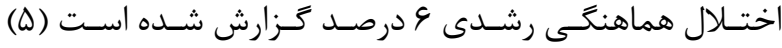

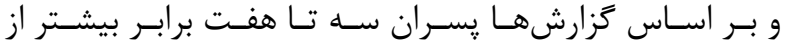

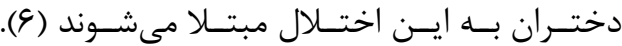

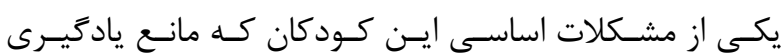

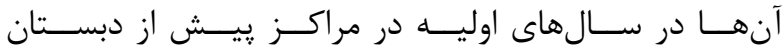

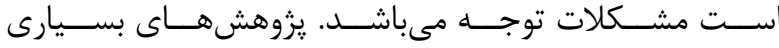

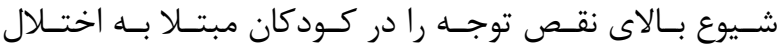

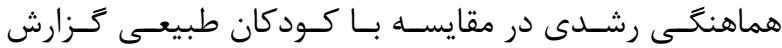

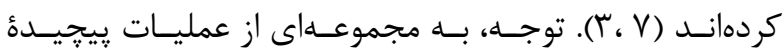

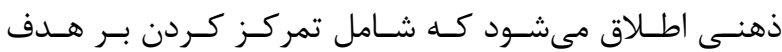

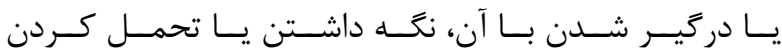

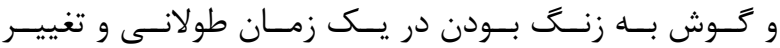

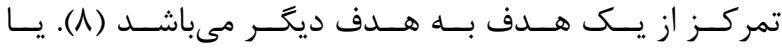

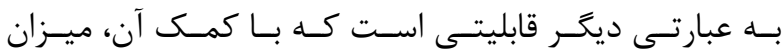

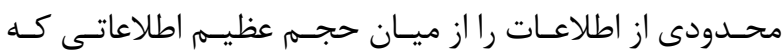

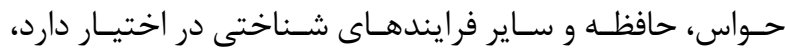
بــه صـورت فعـال يـردازش مى كنــــ (9).

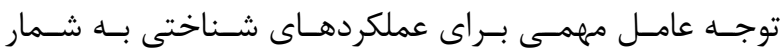

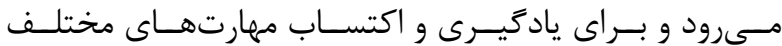

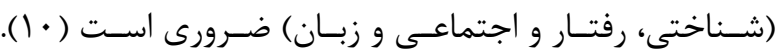

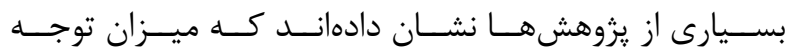

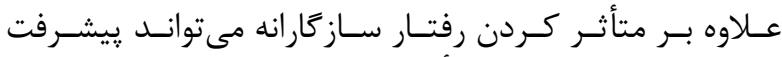

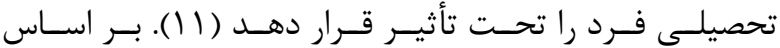

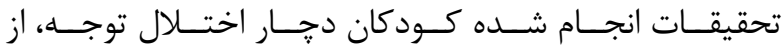

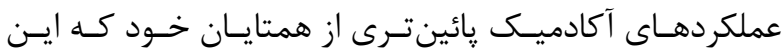

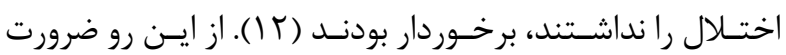

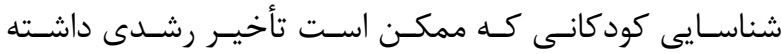

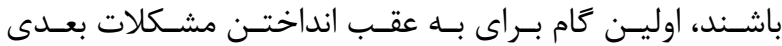

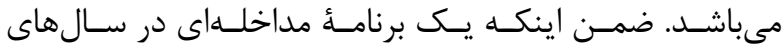

${ }^{4}$ Audiffren

${ }^{5}$ Fragala-Pinkham, Haley and O’Neil 
ابزارهاى مورد استفاده در تحقيق

الف - سياهؤ اختلال هماهنكى رشدى ويلسون

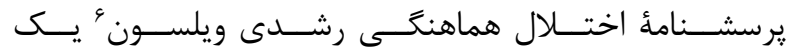

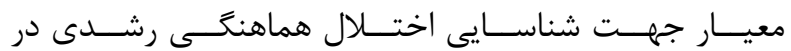

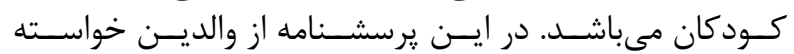

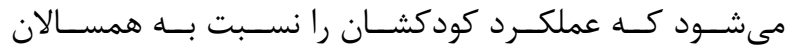

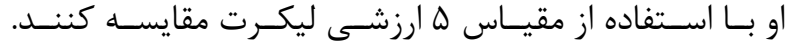

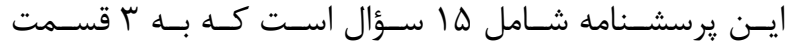

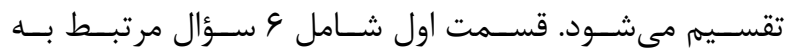

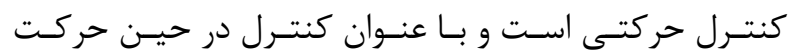

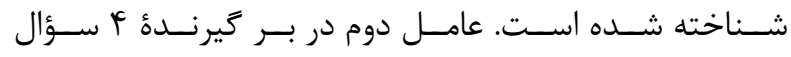

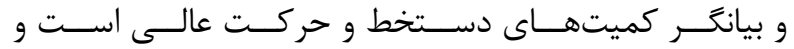

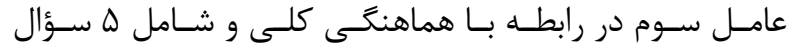

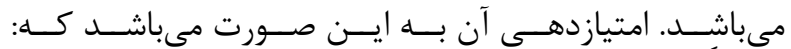

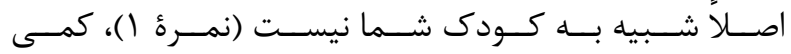

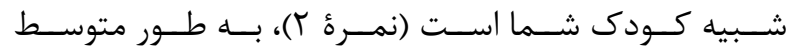

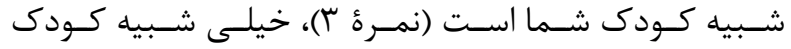

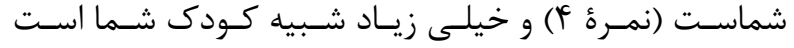

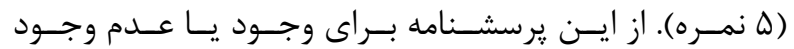

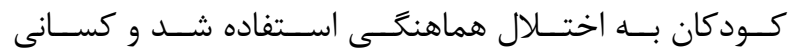

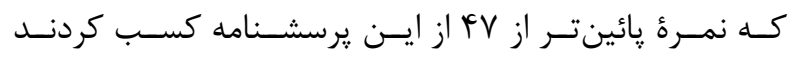

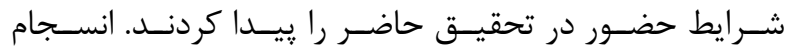

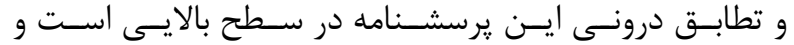

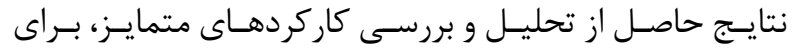

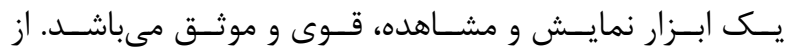

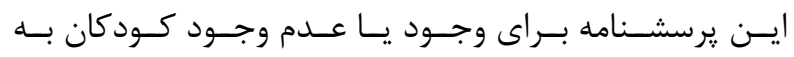

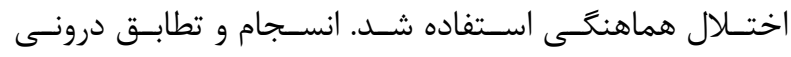

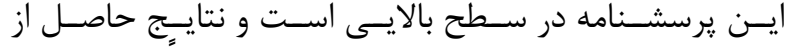

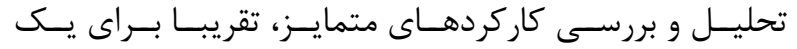

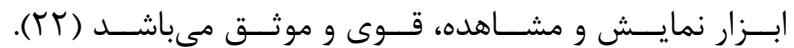

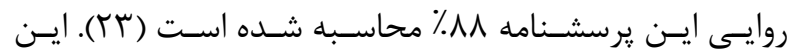

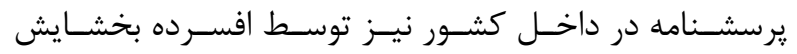

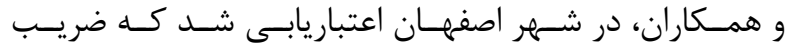

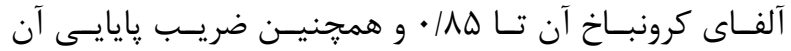

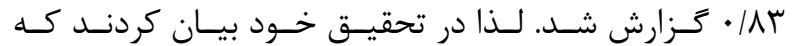

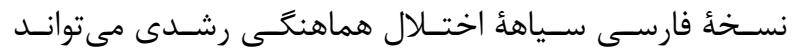

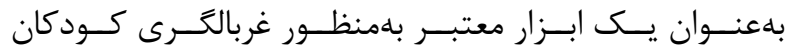

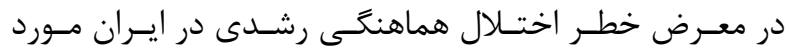

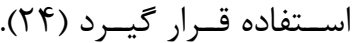

$$
\text { ب- ب - آزمون كانرز }
$$

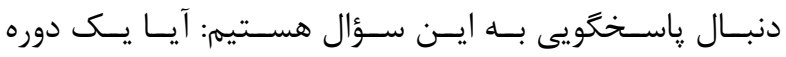

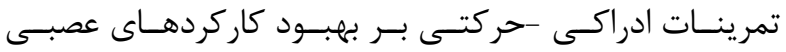

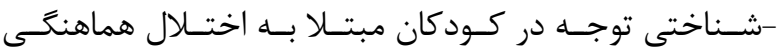

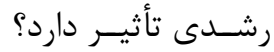

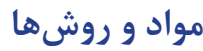

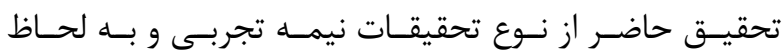

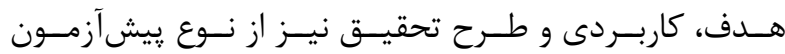

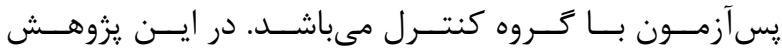

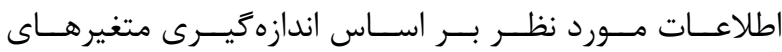

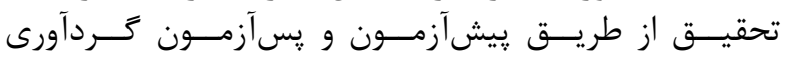

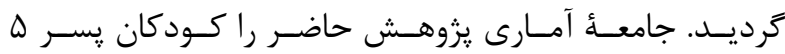

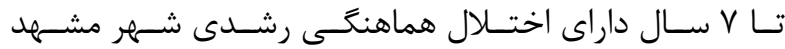

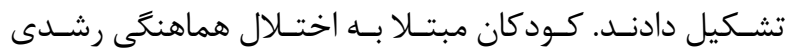

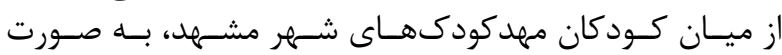

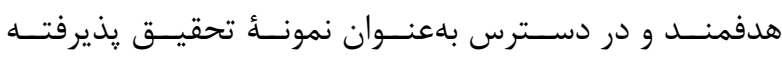

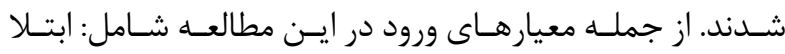

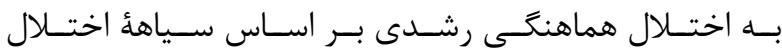

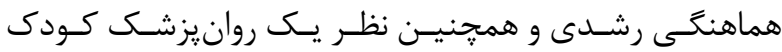

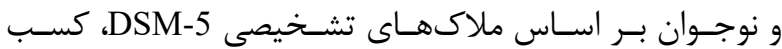

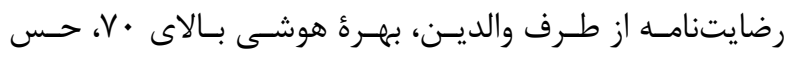

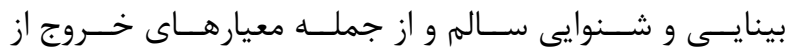

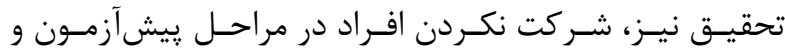

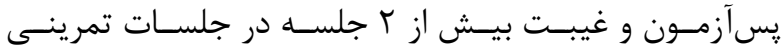

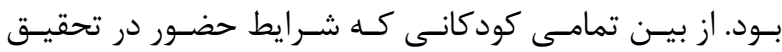

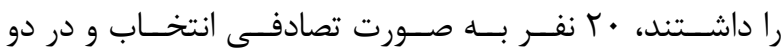

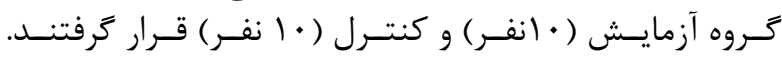

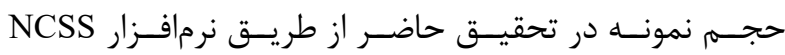

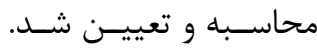

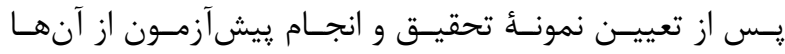

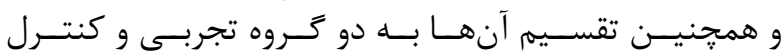

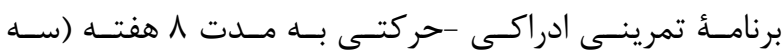

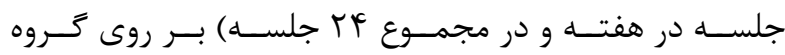

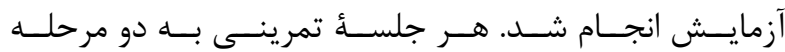

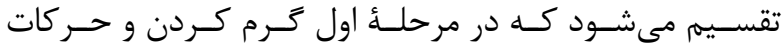

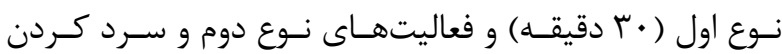

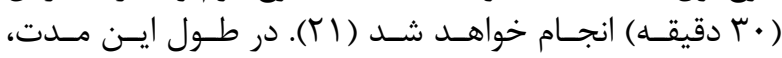

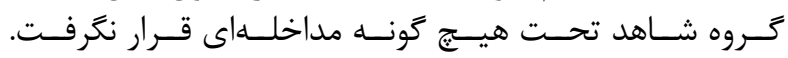

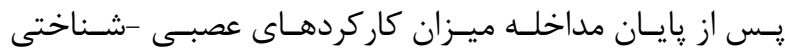

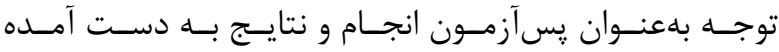

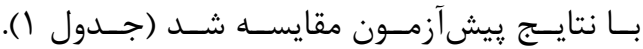

\begin{tabular}{|c|c|c|c|}
\hline يس از مداخله (YF جلسه) & مداخله & قبل از مداخله & 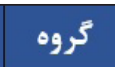 \\
\hline ارزيابى كاركردهاى عصبى -شناختى توجه & فعاليتهاى ادراكى -حركتى & ارزيابى كاركردهاى عصبى -شناختى توجه & 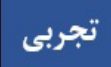 \\
\hline ارزيابى كاركردهاى عصبى -شناختى توجه & ------- & ارزيابى كاركردهاى عصبى -شناختى توجه & 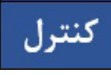 \\
\hline
\end{tabular}




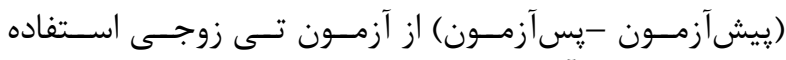

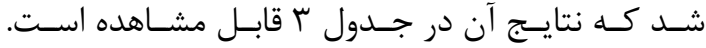

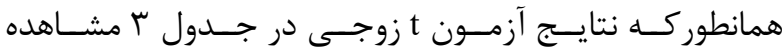

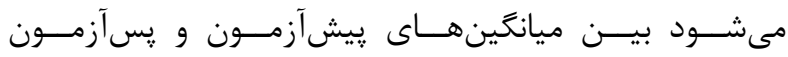

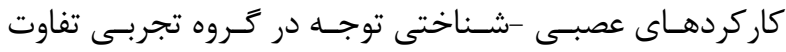

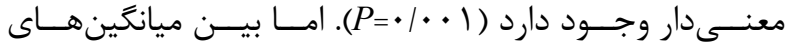

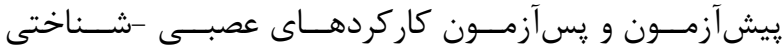

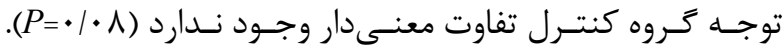

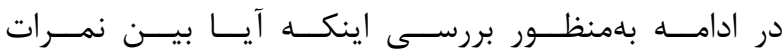

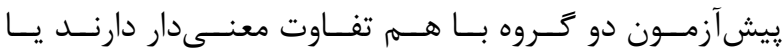

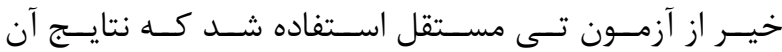

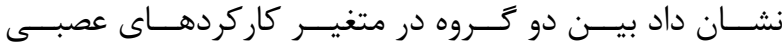

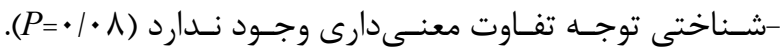

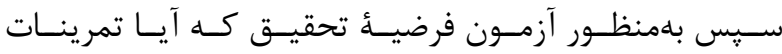

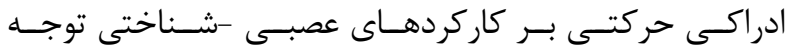

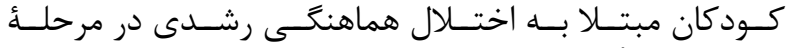

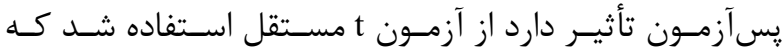

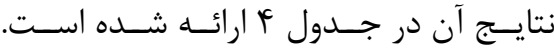

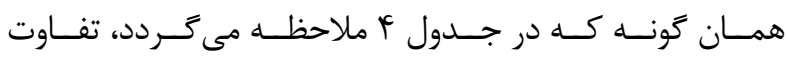

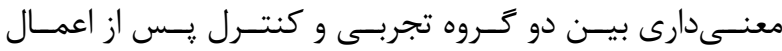

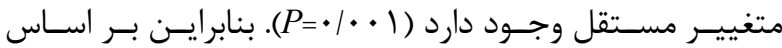

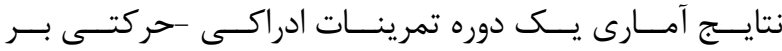

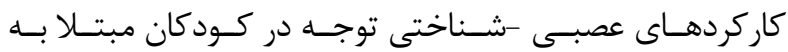

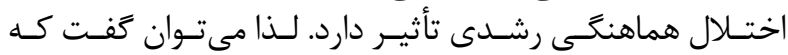

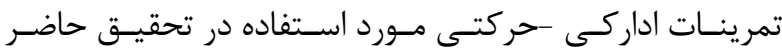

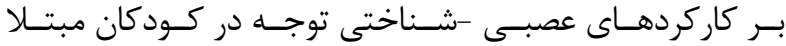

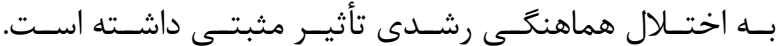

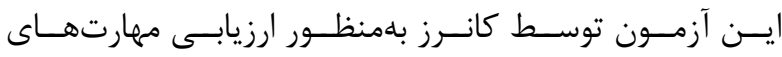

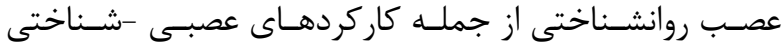

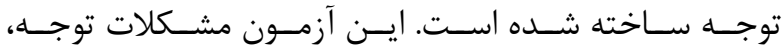

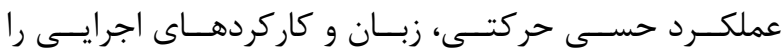

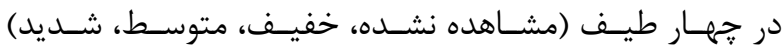

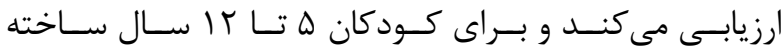

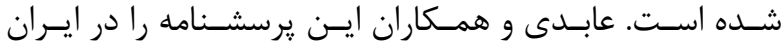

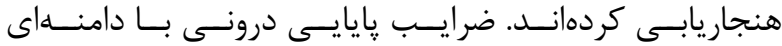

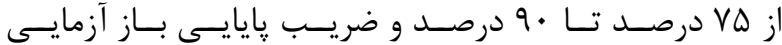

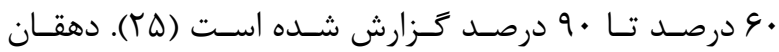

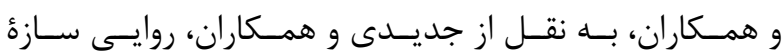

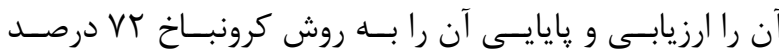

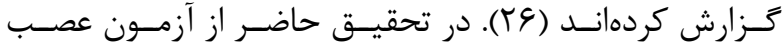

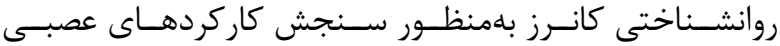

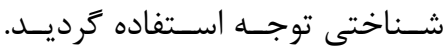

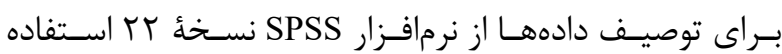

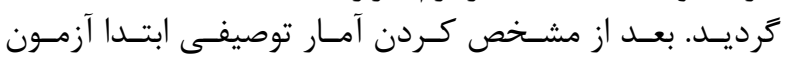

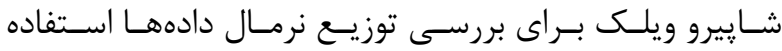

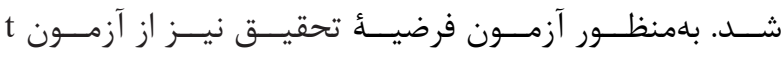

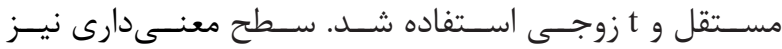

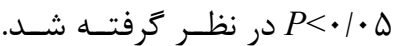

يافتهها

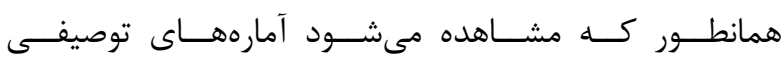

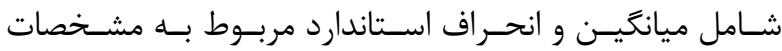

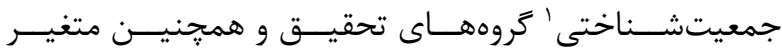

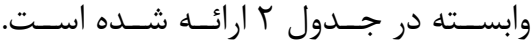

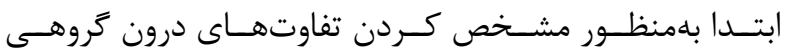

جدول r- يِيشآزمون و يسآزمون متغيرهاى تحقيق و مشخصات شركت كندكان در كروههاى كنترل و مداخله.

\begin{tabular}{|c|c|c|c|}
\hline 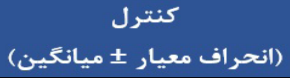 & 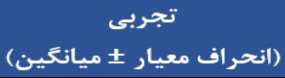 & \multicolumn{2}{|r|}{ 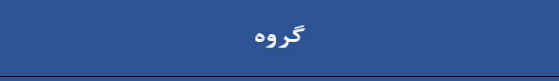 } \\
\hline •ا نفر & •ا نفر & \multicolumn{2}{|r|}{ تعداد } \\
\hline$\Delta / A \vee \pm 1 / . \varphi$ & $\Delta / \& T \pm 1 / .9$ & \multicolumn{2}{|r|}{ سن (سال) } \\
\hline $9 r / V \cdot \pm 1 \cdot / 99$ & $9 r / 1 \cdot \pm 11 / 9 \wedge$ & \multicolumn{2}{|r|}{ IQ } \\
\hline $10 / r+ \pm r / 18$ & $10 / 9+ \pm r / r r$ & بيشى آزمون & \multirow{2}{*}{ كاركردهاى عصبى -شناختى توجه } \\
\hline $10 / 8 \cdot \pm r / 9 \Delta$ & $9 / \lambda+ \pm \mid / \wedge)$ & يس آزمون & \\
\hline
\end{tabular}

\begin{tabular}{|c|c|c|c|c|}
\hline معنى سطارى & مقدار t & عمبر - شناخ كاركردهاى توجه & مرحله & كروه \\
\hline \multirow{2}{*}{$\cdot / \cdot \wedge$} & \multirow{2}{*}{$1 / 98$} & $10 / 2 \cdot \pm 4 / 18$ & بيشى آزمون & \multirow{2}{*}{ كنترل } \\
\hline & & $1 \Delta / 8 \cdot \pm T / 9 \Delta$ & "ِس آزمون & \\
\hline \multirow{2}{*}{$\cdot 1+\cdots 1$} & \multirow{2}{*}{$\Delta / \Delta F$} & $10 / 9 \cdot \pm r / V T$ & بيش آزمون & \multirow{2}{*}{ تجربى } \\
\hline & & $9 / \wedge \cdot \pm / / \wedge /$ & يسى آزمون & \\
\hline
\end{tabular}

\begin{tabular}{|c|c|c|c|c|}
\hline سطح معنى دارى & df & مقدار t & مرحله & \multirow{3}{*}{ كاركردهاى عصبى -شناختى توجه } \\
\hline.$/ v$ & 11 & +/r人 & يِيش آزمون & \\
\hline$+1++1 *$ & in & $\Delta / r_{q}$ & يسى آزمون & \\
\hline
\end{tabular}

${ }^{7}$ Demographic 


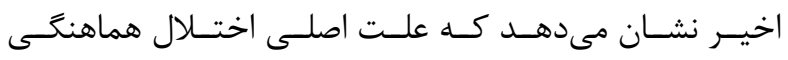

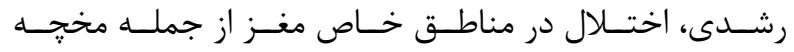

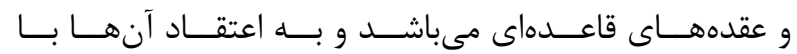

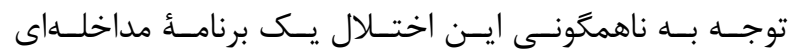

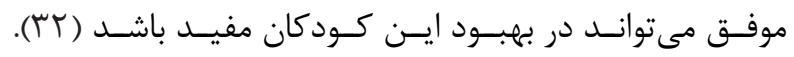

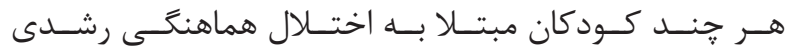

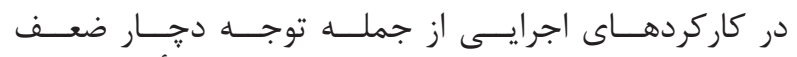

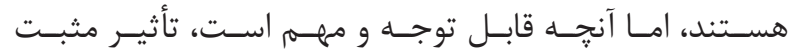

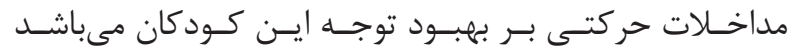

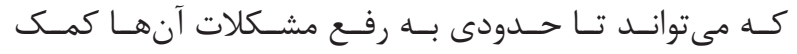

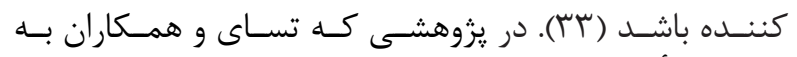

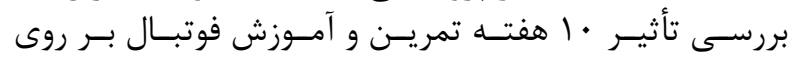

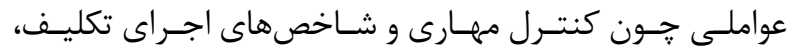

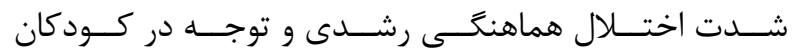

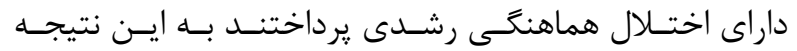

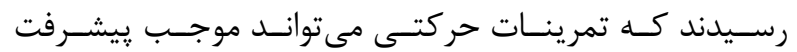

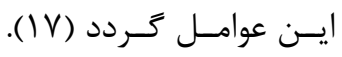

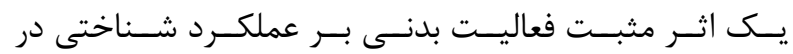

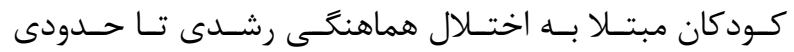

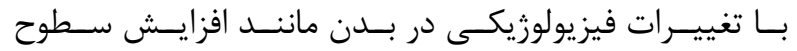

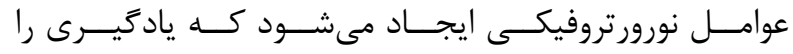

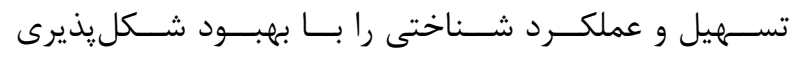

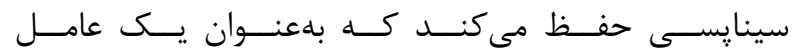

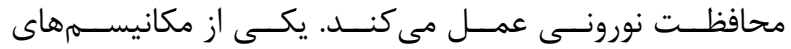

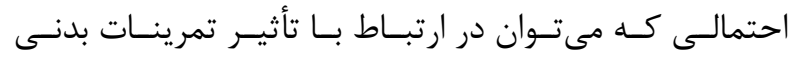

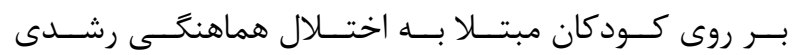

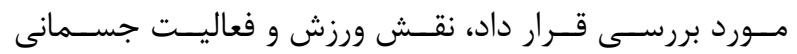

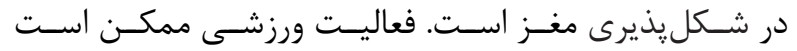

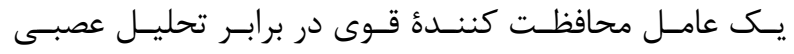

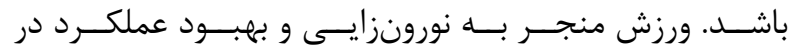

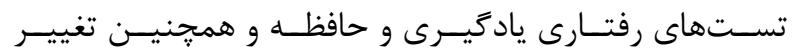

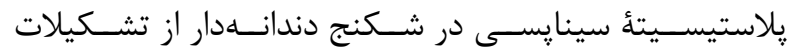

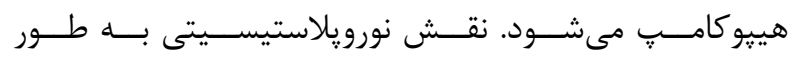

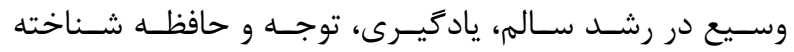

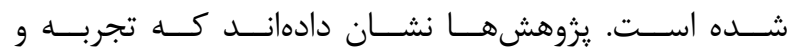

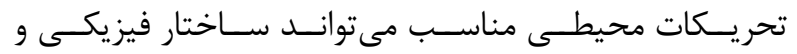

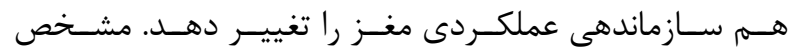

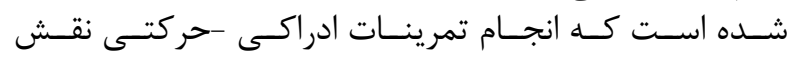

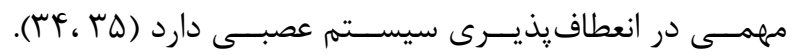

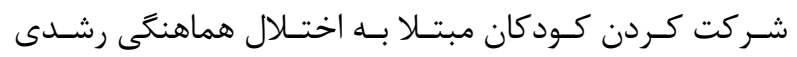

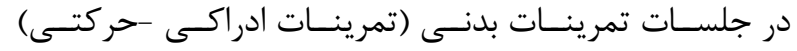

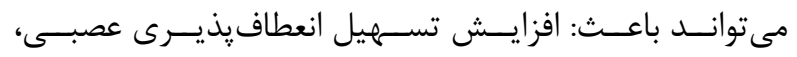

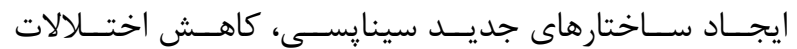

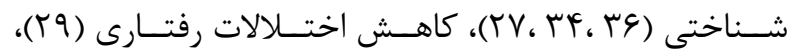

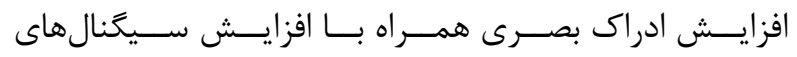

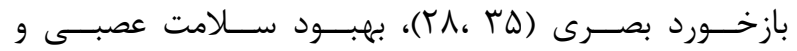

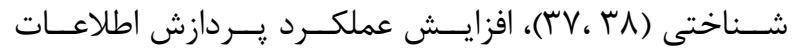

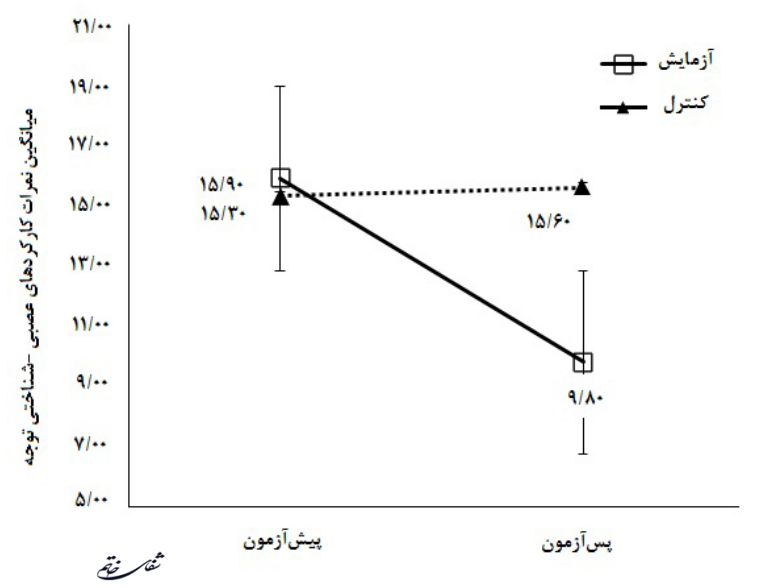

نمــودار ا - ميانكيـن نمـرات كاركردهـاى عصبـى -شــناختى توجـهـ در مراحـل

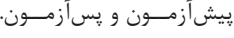

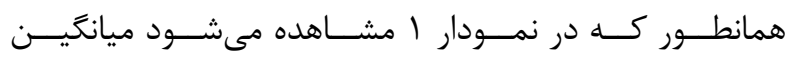

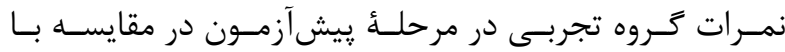

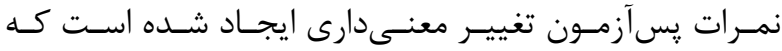

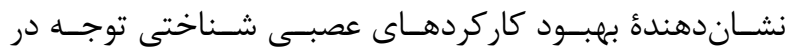

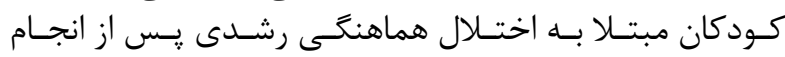

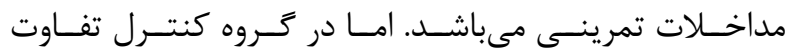

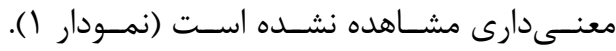
بحث و نتيجه كيرى

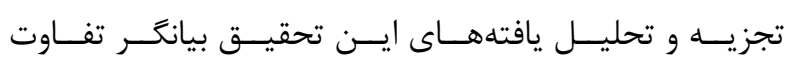

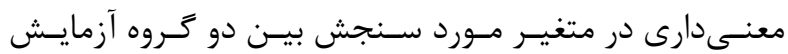

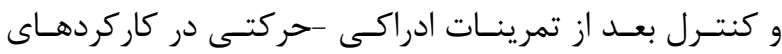

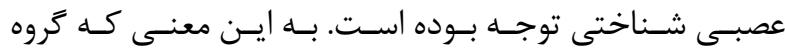

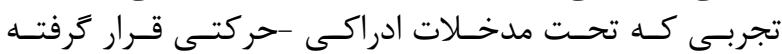

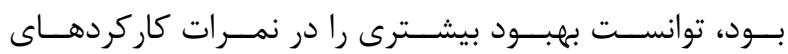

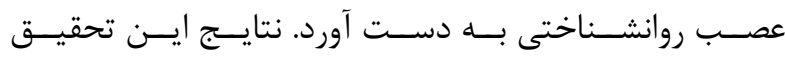

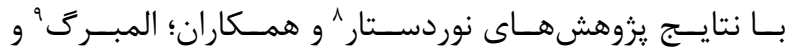

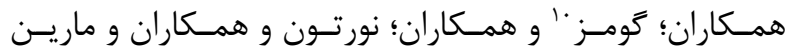

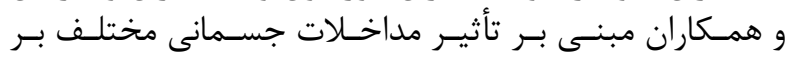

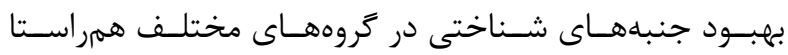

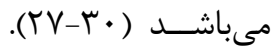

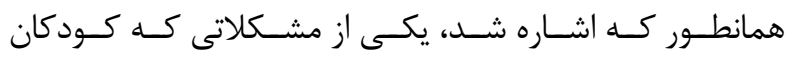

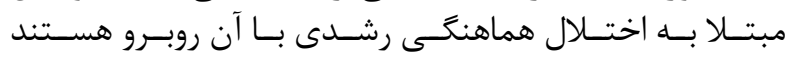

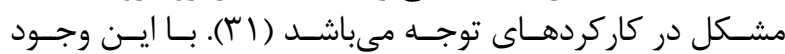

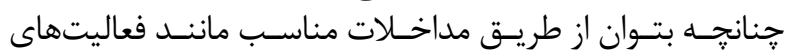

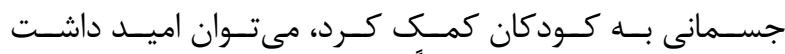

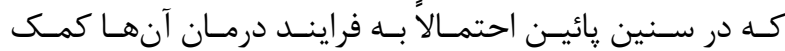

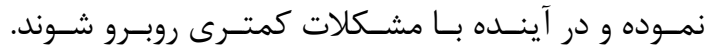

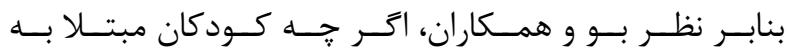

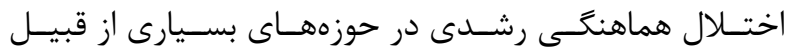

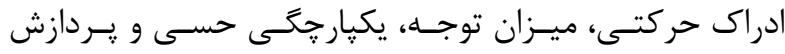

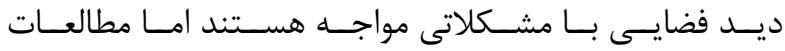




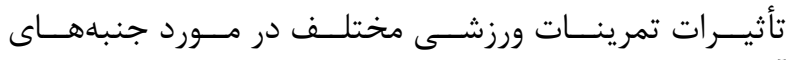

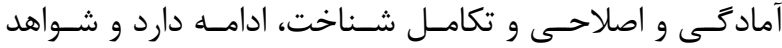

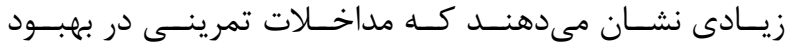

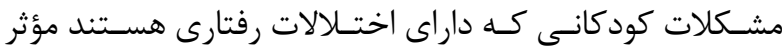

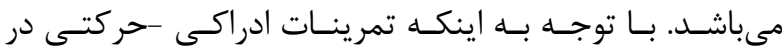

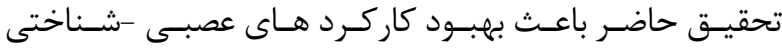

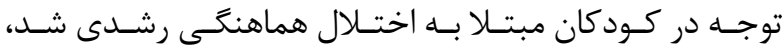

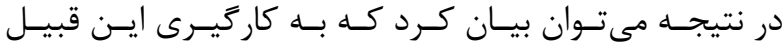

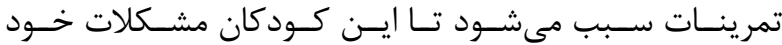

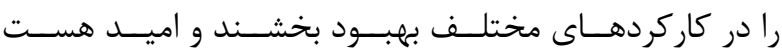

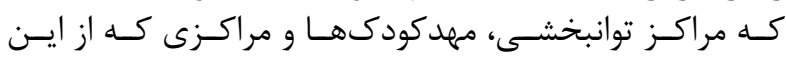

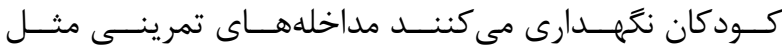

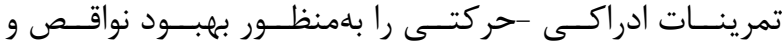

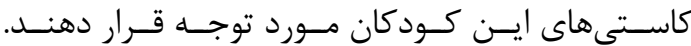

$$
\text { تشكر و قدردانى }
$$

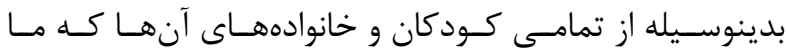

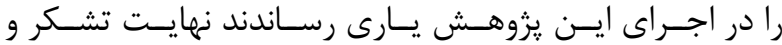

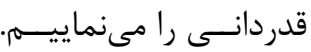

1. Payne VG, Isaacs LD. Human motor development: a lifespan approach. $8^{\text {th }}$ ed. McGraw-Hill Education. 2017. p. 608.

2. Halperin JM, Healey DM. The influences of environmental enrichment, cognitive enhancement, and physical exercise on brain development: Can we alter the developmental trajectory of ADHD? Neurosci Biobehav Rev. 2011; 35(3): 621-34.

3. Sugden D. Current approaches to intervention in children with developmental coordination disorder. Dev Med Child Neurol. 2007; 49(6): 467-71.

4. American Psychiatric Association. Diagnostic and statistical manual of mental disorders (DSM-5®). Am J Psychiatr. 2013; p. 947.

5. Wuang YP, SU JH, SU CY. Reliability and responsiveness of the movement assessment battery for children-second edition test in children with developmental coordination disorder. Dev Med Child Neurol. 2012; 54(2): 160-5.

6. Kadesjo B, Gillberg C. Developmental coordination disorder in Swedish 7-year-old children. J Am Acad Child Adolesc Psychiatr. 1999; 38(7): 820-8.

7. Kaplan BJ, Wilson BN, Dewey D, Crawford SG. DCD may not be a discrete disorder. Hum Mov Sci.

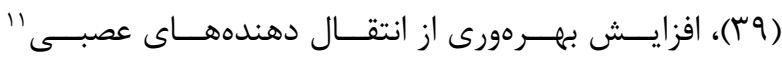

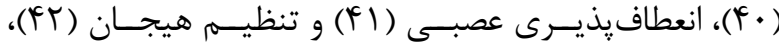

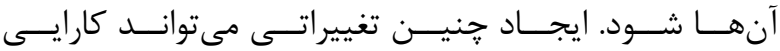

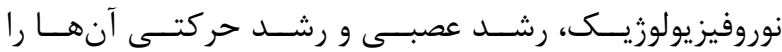
بهبــود ببخشـنـد.

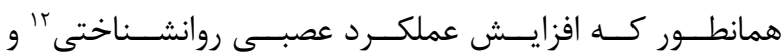

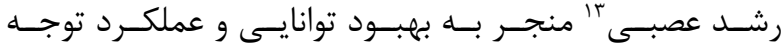

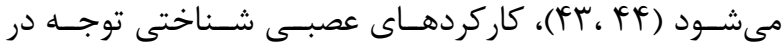

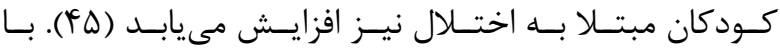

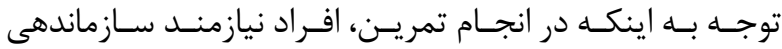

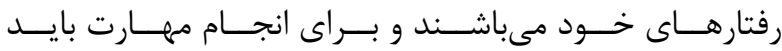

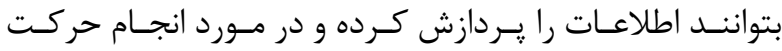

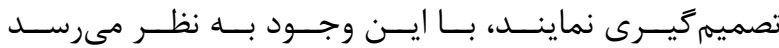

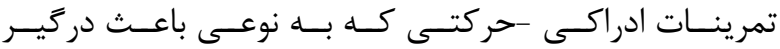

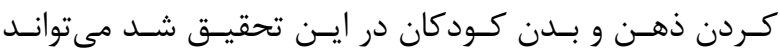

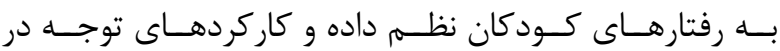

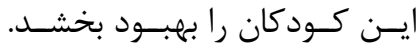
بهعنـوان جمعبنــدى كلـى، تحقيقــات بـراى مســتند كـردن

منابع

1998; 17(4-5): 471-90.

8. Seidman LJ. Neuropsychological functioning in people with ADHD across the lifespan. Clin Psychol Rev. 2006; 26(4): 466-85.

9. Sternberg RJ, Sternberg K. Cognitive psychology. $6^{\text {th }}$ ed. Nelson Education. 2016.

10. Rezaiyan A, Mohammadi E, Fallah PA. Effect of computer game intervention on the attention capacity of mentally retarded children. Int J Nurs Sci Pract. 2007; 13(5): 284-8.

11. Crimmens P. Drama therapy and storymaking in special education. Jessica Kingsley Publishers. 2006; p. 208.

12. Barkley RA, Murphy KR. Attention-deficit hyperactivity disorder: A clinical workbook. $2^{\text {nd }}$ ed. Guilford Press; p 134. 1998.

13. Venetsanou F, Kambas A, Aggeloussis N, Fatouros I, Taxildaris K. Motor assessment of preschool aged children: A preliminary investigation of the validity of the Bruininks-oseretsky test of motor proficiency-Short form. Hum Mov Sci. 2009; 28(4): 543-50.

14. Davarmanesh A, Baratysadeh F. Precedent of handicaps rehabilitation principles. Tehran Roshd publication. 2006: p. 169-81.

${ }^{11}$ Neurotransmitter

12 Neurophysiological

13 Neurodevelopmental 


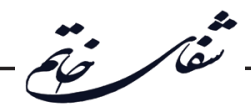

15. Jane JJ, Sit CH, Burnett AF. Motor skill interventions in children with developmental coordination disorder: A systematic review and meta-analysis. Arch Phys Med Rehabil. 2018; 99(10): 2076-99.

16. Smits-Engelsman BC, Jelsma LD, Ferguson GD. The effect of exergames on functional strength, anaerobic fitness, balance and agility in children with and without motor coordination difficulties living in low-income communities. Hum Mov Sci. 2017; 55: 327-37.

17. Tsai C-L, Wang C-H, Tseng Y-T. Effects of exercise intervention on event-related potential and task performance indices of attention networks in children with developmental coordination disorder. Brain Cogn. 2012; 79(1): 12-22.

18. Tomporowski PD, Ganio MS. Short-term effects of aerobic exercise on executive processing, memory, and emotional reactivity. Int J Sport Psychol. 2006; 4(1): 57-72.

19. Audiffren M, Tomporowski PD, Zagrodnik J. Acute aerobic exercise and information processing: modulation of executive control in a Random Number Generation task. Acta Psychol. 2009; 132(1): 85-95.

20. Fragala-Pinkham M, Haley SM, O’Neil ME. Group aquatic aerobic exercise for children with disabilities. Dev Med Child Neurol. 2008; 50(11): 822-7.

21. Moradi H, Khodashenas E, Sohrabi M, Teymoori S, Shayan-Noshabadi A. The effect of Spark motor program on sensory-motor functions in children with developmental coordination disorder. Feyz Journal of Kashan University of Medical Sciences. 2015; 19(5): 391-8.

22. Wilson PH. Practitioner review: approaches to assessment and treatment of children with DCD: an evaluative review. J Child Psychol Psychiatry. 2005; 46(8): 806-23.

23. Wilson BN, Crawford SG, Green D, Roberts G, Aylott A, Kaplan BJ. Psychometric properties of the revised developmental coordination disorder questionnaire. Phys Occup Ther Pediatr. 2009; 29(2): 182-202.

24. Afsordeh Bakhshayesh R, Salehi H, Movahedi A, Ghasemi V. The log version of the psychometric properties of coordination impaired motor development in children 6-11 years old. Journal of Psychology Special. 2010; 1(4): 135-61.

25. Abedi A, Malekpour MP, Moulavi H, Arizihr Amiri $\mathrm{SH}$. Compare neuropsychological features young children with neuropsychological/ development learning disabilities and normal, preschool. Res Exceptional Children. 2007; 2(10): 38-48.

26. Dehghan M, Karimei N, Tagipourjavan AA, Hasan NattajJelodar F, Zaidabadi F. The effectiveness of rhythmic movement games (weighted) on the rate of executive function in children with neuropsychological learning disabilities. J Learning Disabil. 2012; 2(1/5377).

27. Ellemberg D, St-Louis-Deschênes M. The effect of acute physical exercise on cognitive function during development. Psychol Sport Exerc. 2010; 11(2): 122-6.

28. Norton DJ, McBain RK, Öngür D, Chen Y. Perceptual training strongly improves visual motion perception in schizophrenia. Brain Cognit. 2011; 77(2): 248-56.

29. Marin R, Williams A, Hale S, Burge B, Mense $\mathrm{M}$, Bauman R, et al. The effect of voluntary exercise exposure on histological and neurobehavioral outcomes after ischemic brain injury in the rat. Physiol Behav. 2003; 80(2-3): 167-75.

30. Noordstar JJ, van der Net J, Voerman L, Helders PJ, Jongmans MJ. The effect of an integrated perceived competence and motor intervention in children with developmental coordination disorder. Res Dev Disabil. 2017; 60: 162-75.

31. Dewey D, Kaplan BJ, Crawford SG, Wilson BN. Developmental coordination disorder: associated problems in attention, learning, and psychosocial adjustment. Hum Mov Sci. 2002; 21(5-6): 905-18.

32. Bo J, Lee C-M. Motor skill learning in children with developmental coordination disorder. Res Dev Disabil. 2013; 34(6): 2047-55.

33. Ball MF. Developmental coordination disorder: hints and tips for the activities of daily living. Jessica Kingsley Publishers. 2002; p. 98.

34. Gomez-Pinilla F. The combined effects of exercise and foods in preventing neurological and cognitive disorders. Prev Med. 2011; 52: S75-S80.

35. Pourtois G, Rauss KS, Vuilleumier P, Schwartz S. Effects of perceptual learning on primary visual cortex activity in humans. Vis Res. 2008; 48(1): 55-62.

36. Radak Z, Kumagai S, Taylor AW, Naito H, Goto S. Effects of exercise on brain function: role of free radicals. Appl Physiol Nutr Metabol. 2007; 32(5): 942-6.

37. Uysal N, Tugyan K, Kayatekin BM, Acikgoz O, Bagriyanik HA, Gonenc S, et al. The effects of regular 
aerobic exercise in adolescent period on hippocampal neuron density, apoptosis and spatial memory. Neurosci Lett. 2005; 383(3): 241-5.

38. Velikonja O, Čurić K, Ožura A, Jazbec SŠ. Influence of sports climbing and yoga on spasticity, cognitive function, mood and fatigue in patients with multiple sclerosis. Clin Neurol Neurosurg. 2010; 112(7): $597-$ 601.

39. Audiffren M, Tomporowski PD, Zagrodnik J. Acute aerobic exercise and information processing: energizing motor processes during a choice reaction time task. Acta Psychol. 2008; 129(3): 410-9.

40. Leung L-Y, Tong K-Y, Zhang S-M, Zeng X-H, Zhang K-P, Zheng X-X. Neurochemical effects of exercise and neuromuscular electrical stimulation on brain after stroke: a microdialysis study using rat model. Neurosci Lett. 2006; 397(1-2): 135-9.

41. Ploughman M. Exercise is brain food: the effects of physical activity on cognitive function. Dev Neurorehabil. 2008; 11(3): 236-40.

42. Kaplan HI, Sadock BJ. Kaplan and Sadock's synopsis of psychiatry: behavioral sciences/clinical psychiatry. LWW; Tenth, North American edition. 1998.

43. Eimer M, Forster B, Van Velzen J, Prabhu G. Covert manual response preparation triggers attentional shifts: ERP evidence for the premotor theory of attention. Neuropsychologia. 2005; 43(6): 957-66.

44. Eimer M, Van Velzen J, Gherri E, Press C. Manual response preparation and saccade programming are linked to attention shifts: ERP evidence for covert attentional orienting and spatially specific modulations of visual processing. Brain Res. 2006; 1105(1): 7-19.

45. Afshari J. The effect of perceptual-motor training on attention in the children with autism spectrum disorders. Res Autism Spectr Disord. 2012; 6(4): 1331-6. 